\title{
Study of the particle transverse momentum spectra in relativistic heavy ion collisions using the Tsallis statistics
}

\author{
Oana Ristea $^{1 \mathrm{a}}$, Alexandru Jipa ${ }^{1}$, Catalin Ristea ${ }^{1}$, Calin Besliu ${ }^{1}$, Ionel Lazanu ${ }^{1}$, Marius Calin ${ }^{1}$, Tiberiu \\ Esanu $^{1}$ and Vanea Covlea ${ }^{1}$ \\ ${ }^{1}$ Atomic and Nuclear Physics Chair, Department of Structure of Matter, Atmosphere and Earth \\ Physics, Astrophysics, Faculty of Physics, University of Bucharest, Romania
}

\begin{abstract}
In this work, we have analized the transverse momentum spectra of charged pions produced in $\mathrm{Au}+\mathrm{Au}$ collisions at different energies using the non-extensive Tsallis distribution. The centrality and rapidity dependence of the non-extensivity parameter, q, is studied. In addition, comparisons with simulated heavy ion collisions data using the UrQMD code will be presented.
\end{abstract}

\section{Introduction}

High-energy heavy ion collisions provide an unique opportunity to study the nuclear matter under extreme conditions. Due to the huge multiplicities of hadrons created in A+A collisions at relativistic energies, the statistical approach is suitable to describe the particle production. However, it is possible that the produced matter may be formed in a state which is far away from the local equilibrium, because immediately after the collision the system starts to expands in both longitudinal and transverse direction.

Recently, many authors have used Tsallis statistics to characterize the particle production in high energy nuclear collisions [1-3]. This statistics is appropriate for the study of complex systems with a certain degree of non-equilibrium as can be the case with these nuclear collisions. The transverse momentum spectra can be described by this Tsallis distribution [4] characterized by a non-extensivity parameter q:

$$
h_{q}\left(p_{T}\right)=C_{q}\left[1-(1-q){\frac{p_{T}}{T}}^{\frac{1}{1-q}}\right.
$$

where $\mathrm{C}_{\mathrm{q}}$ is a normalization constant and $\mathrm{q}$ is a parameter describing the degree of non-equilibrium of the produced system. The Tsallis distribution can be considered as a generalization of the usual exponential Boltzmann-Gibbs (BG) distribution and converges to it when the parameter $\mathrm{q}$ tends to unity:

$$
h\left(p_{T}\right) \Rightarrow^{q \rightarrow 1} C_{1} \exp \left(-\frac{p_{T}}{T}\right)
$$

The non-extensivity parameter, $\mathrm{q}$, is correlated with the presence of temperature fluctuations inside the fireball (the temperature of the fireball fluctuates from event to event or also in the same event) These fluctuations cause the system to depart from equilibrium. If the nonextensivity parameter $\mathrm{q}=1$,

\footnotetext{
${ }^{a}$ Corresponding author: oana@brahms.fizica.unibuc.ro
} 
there are no temperature fluctuations and a value $q>1$ indicates presence of fluctuations. These fluctuations could be caused mainly by the possible energy transfer between the central fireball (participants) and nuclear fragments passing by without interaction (spectators).

However, it must be emphasized that in heavy ion collisions there could be other sources for temperature fluctuations, such as effects of resonance decays, kinetic non-equilibrium in the dynamics also for the average trajectory in the phase-space.

\section{Results}

Transverse momentum spectra of charged pions produced in $\mathrm{Au}+\mathrm{Au}$ collisions at $\sqrt{s_{N N}}=200$ $\mathrm{GeV}$ and measured by the BRAHMS experiment [5,6] were fitted using formula 1 and the fit parameter (q-1) is shown in Figure 1 as a function of the number of participants. The non-extensivity parameter is smaller in central $\mathrm{Au}+\mathrm{Au}$ collisions and increases going to peripheral collisions. The results suggest that the degree of non-equilibrium is higher in peripheral collisions. The centrality dependence of the $(\mathrm{q}-1)$ parameter suggests an evolution from a non-equilibrated system in peripheral $\mathrm{Au}+\mathrm{Au}$ collisions towards a more thermalized system in central $\mathrm{Au}+\mathrm{Au}$ collisions.

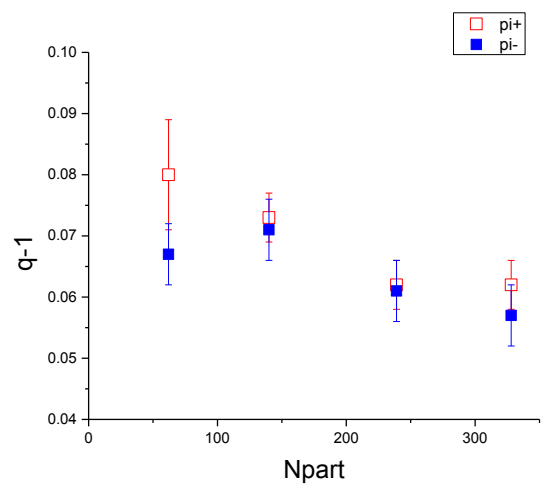

Figure 1. The centrality dependence of the non-extensivity parameter obtained from fits to charged pion pt spectra in $\mathrm{Au}+\mathrm{Au}$ collisions at $\sqrt{S_{N N}}=200 \mathrm{GeV}$.

Comparing the results obtained by fitting the $\mathrm{p}_{\mathrm{T}}$ spectra of charged pions produced in the most central $0-10 \% \mathrm{Au}+\mathrm{Au}$ collisions at $\sqrt{S_{N N}}=200 \mathrm{GeV}$ and $62.4 \mathrm{GeV}$, we observe that the non-extensivity parameter values are approximately equals for the two energies, but the temperatures are higher for the top RHIC energy (Table 1).

Table 1. The system temperature, $\mathrm{T}$, and the non-extensivity parameter, $\mathrm{q}-1$, obtained in $0-10 \% \mathrm{Au}+\mathrm{Au}$ collisions at $\sqrt{S_{N N}}=62.4 \mathrm{GeV}$ and $200 \mathrm{GeV}$.

\begin{tabular}{|c|c|c|c|c|}
\hline$\sqrt{S_{N N}}[\mathrm{GeV}]$ & $\mathrm{T}_{\pi^{+}}[\mathrm{MeV}]$ & $(\mathrm{q}-1)_{\pi^{+}}$ & $\mathrm{T}_{\pi-}[\mathrm{MeV}]$ & $(\mathrm{q}-1)_{\pi-}$ \\
\hline 200 & $186.3 \pm 3.9$ & $0.062 \pm 0.004$ & $187.5 \pm 4.5$ & $0.057 \pm 0.005$ \\
\hline 62.4 & $167.2 \pm 6.5$ & $0.063 \pm 0.008$ & $161.8 \pm 4.2$ & $0.066 \pm 0.005$ \\
\hline
\end{tabular}

We analized the $\pi^{-}$transverse momentum spectra obtained in $\mathrm{Au}+\mathrm{Au}$ collisions at $\sqrt{S_{N N}}=62.4$ $\mathrm{GeV}$, for different rapidities ( $\mathrm{y}=0,0.8,1,3.1$ and 3.2). Figure 2 presents the degree of agreement between the experimental data and the Tsallis fits with Eq.1 (red lines). 

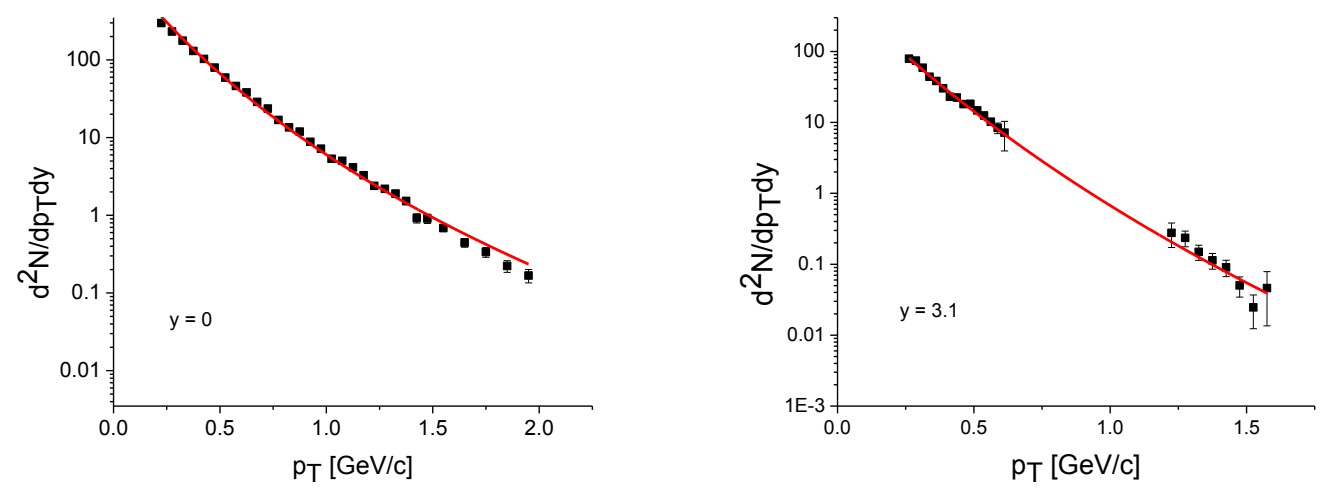

Figure 2. Transverse momentum spectra for negative pions produced in $\mathrm{Au}+\mathrm{Au}$ collisions at $\sqrt{s_{N N}}=62.4 \mathrm{GeV}$ at midrapidity, $\mathrm{y}=0$ (left) and at forward rapidity, $\mathrm{y}=3.1$ (right). Data are taken from ref. [7]. Red lines are the fits with Tsallis distribution (Eq. 1).

Table 2 shows the obtained Tsallis fit results. In the rapidity interval $[0,1]$, the non-extensivity parameter and the temperature are almost constant within errors. At forward rapidity, the temperature decreases strongly and $\mathrm{q}$ is increasing. The high value of $\mathrm{q}$ is correlated with high fluctuations in temperature. This is an indication that the system with a higher fluctuation in temperature is far away from its thermal equilibrium.

Table 2. The system temperature, $\mathrm{T}$, and the non-extensivity parameter, $\mathrm{q}-1$, obtained in $\mathrm{Au}+\mathrm{Au}$ collisions at $\sqrt{S_{N N}}=62.4 \mathrm{GeV}$, as a function of rapidity.

\begin{tabular}{|c|c|c|c|c|}
\hline Rapidity & 0 & 0.8 & 1 & 3.1 \\
\hline $\mathrm{T}[\mathrm{MeV}]$ & $161.8 \pm 4.2$ & $156.2 \pm 4.6$ & $163.6 \pm 2.3$ & $110.5 \pm 11.6$ \\
\hline $\mathrm{q}-1$ & $0.066 \pm 0.005$ & $0.072 \pm 0.006$ & $0.063 \pm 0.003$ & $0.076 \pm 0.017$ \\
\hline
\end{tabular}

Transverse momentum spectra of charged pions in simulated $\mathrm{Au}+\mathrm{Au}$ collisions at various energies $\left(\mathrm{p}_{\mathrm{lab}}=5 \mathrm{GeV} / \mathrm{c}, 11 \mathrm{GeV} / \mathrm{c}, 25 \mathrm{GeV} / \mathrm{c}\right.$ and $\left.\sqrt{S_{N N}}=62.4 \mathrm{GeV}, 200 \mathrm{GeV}\right)$ using the UrQMD (UltraRelativistic Quantum Molecular Dynamics) code [8] were fitted using formula 1 considering different system temperatures. The energy dependence of the fit parameter (q-1) for positive pions produced in simulated $\mathrm{Au}+\mathrm{Au}$ collisions is shown in Table 3.

Table 3. The nonextensivity parameter, q-1, for positive pions in $\mathrm{Au}+\mathrm{Au}$ collisions simulated with UrQMD code.

\begin{tabular}{|c|c|c|c|c|c|}
\hline $\mathrm{T}[\mathrm{MeV}]$ & $p_{\mathrm{lab}}=5 \mathrm{AGeV}$ & $p_{\mathrm{lab}}=11 \mathrm{AGeV}$ & $\mathrm{p}_{\mathrm{lab}}=25 \mathrm{AGeV}$ & $\sqrt{S_{N N}}=62.4 \mathrm{GeV}$ & $\sqrt{S_{N N}}=200 \mathrm{GeV}$ \\
\hline 140 & $0.115 \pm 0.024$ & $0.119 \pm 0.028$ & $0.140 \pm 0.041$ & $0.211 \pm 0.039$ & $0.265 \pm 0.028$ \\
\hline 160 & $0.077 \pm 0.028$ & $0.091 \pm 0.023$ & $0.111 \pm 0.035$ & $0.179 \pm 0.040$ & $0.231 \pm 0.045$ \\
\hline 180 & $0.041 \pm 0.017$ & $0.065 \pm 0.019$ & $0.092 \pm 0.020$ & $0.149 \pm 0.037$ & $0.210 \pm 0.041$ \\
\hline
\end{tabular}


For the simulated UrQMD data, the non-equlibrium parameter q-1 is smaller for a higher system temperature and increases as the energy increase. The simulated (q-1) values as compared to experimental data $\mathrm{Au}+\mathrm{Au}$ collisions from RHIC are higher.

\section{Conclusions}

The q parameter value obtained from Tsallis fits to the charged pion $\mathrm{p}_{\mathrm{T}}$ spectra is smaller in central 0 $10 \% \mathrm{Au}+\mathrm{Au}$ collisions at $\sqrt{S_{N N}}=200 \mathrm{GeV}$ than in peripheral (40-60\% centrality) collisions. For $\mathrm{Au}+\mathrm{Au}$ collisions at $\sqrt{S_{N N}}=62.4 \mathrm{GeV}$, the rapidity dependence of the $\mathrm{q}$ and $\mathrm{T}$ showed that at forward rapidity, the $\mathrm{q}$ is higher than at midrapidity and $\mathrm{T}$ is lower. The higher $\mathrm{q}$ value at forward rapidity $(y=3.1)$ indicates that the pions produced in $62.4 \mathrm{GeV} \mathrm{Au}+\mathrm{Au}$ collisions evolved from a region of the fireball which has not yet reach thermal equilibrium. The simulated UrQMD data for $\mathrm{Au}+\mathrm{Au}$ collisions at different energies show that the q parameter value increases with the collision energy. However, temperature fluctuations in relativistic heavy ion collisions may have other sources that need further investigations.

\section{Acknowledgments}

The work of Oana Ristea and Catalin Ristea was supported by the strategic grant POSDRU/89/1.5/S/58852, Project Postdoctoral programme for training scientific researchers, cofinanced by the European Social Found within the Sectorial Operational Program Human Resources Development 2007-2013. This work was partially supported by PN-II-ID-PCE-IDEI 34/05.10.2011 grant.

\section{References}

1. G. Wilk, Z. Wlodarczyk, Eur. Phys. J. A 40, 299 (2009) ; G. Wilk, Z. Wlodarczyk, Phys. Rev. C 79, 054903 (2009)

2. K. Urmossy, G. G. Barnafoldi, T. S. Biro, Phys.Lett. B 701, 111 (2011)

3. Bhaskar De, S. Bhattacharyya, Goutam Sau, S.K. Biswas, Int.J.Mod.Phys. E 16, 1687 (2007)

4. C. Tsallis, J. Stat. Phys. 52, 479 (1988); Physica A 221, 277 (1995); Braz. J. Phys. 29, 1 (1999); D. Prato and C. Tsallis, Phys. Rev. E 60, 2398 (1999)

5. M. Adamczyk et al. (BRAHMS Collaboration), Nucl. Instr. And Meth. A 499, 437 (2003)

6. I. Arsene et al. (BRAHMS Collaboration), Phys. Rev. C 72, 014908 (2005)

7. I. Arsene et al. (BRAHMS Collaboration), Phys.Lett. B 687, 36 (2010)

8. S.A. Bass et al., Prog. Part. Nucl. Phys. 41, 225 (1998); M. Bleicher et al., J. Phys. G 25, 1859 (1999) 